\title{
Involvement of Rac/Cdc42/PAK pathway in cytoskeletal rearrangements
}

\author{
Joanna Szczepanowska ${ }^{凶}$ \\ Laboratory of Bioenergetics and Biomembranes, Nencki Institute of Experimental Biology, Polish Academy of \\ Sciences, Warszawa, Poland
}

Received: 22 May, 2009; revised: 05 June, 2009; accepted: 08 June, 2009

available on-line: 10 June, 2009

\begin{abstract}
The p21-activated kinases (PAKs) are serine/threonine protein kinases interacting with small GTPases - Rac and Cdc42. PAKs are found in most eukaryotes and play an evolutionarily conserved role in many cellular processes. Six human PAKs have been identified, and based on homology, they can be classified into two groups. This review focuses specifically on the role of Rac/Cdc42 regulated PAKs in maintaining and remodeling cytoskeletal structure in various organisms. A list of PAKs substrates and binding partners implicated directly and indirectly in cytoskeletal reorganization is presented. Also perturbations of the Rac/Cdc42/PAK pathway leading to tumorigenesis and neurodegenerative diseases are reviewed.
\end{abstract}

Keywords: PAK kinase, Rho GTPases, cytoskeletal organization, PAK substrates

\section{INTRODUCTION}

p21-Activated kinases (PAKs) are serine/threonine protein kinases involved in multiple biological processes, but a growing list of data shows that PAKs are mainly implicated in cytoskeletal rearrangements. Cytoskeletal organization and dynamics play a central role in cell movement, migration, adhesion, proliferation, differentiation, and vesicle trafficking.

PAKs were first identified as effectors of small GTPases of the Rho family (Manser et al., 1994). The Rho GTPases exist in two conformations: inactive, GDP-bound, and active, GTP-bound. These proteins are activated by members of the guanine exchange factors family (GEFs) that increase the GDP/GTP ex- change rates, and are inactivated by members of the GTPase activating proteins family (GAPs) (Jaffe \& Hall, 2005). Two Rho GTPases, Rac and Cdc42, can directly interact with PAK.

All PAKs share a conserved catalytic domain located at the C-terminus. PAKs have been found in a broad spectrum of eukaryotic organisms from yeast to humans (for references see: Knaus \& Bokoch, 1998; Hofmann et al., 2004). The six PAK isoforms identified in humans are classified into two groups (I and II) according to the similarity of their catalytic domains, and regulatory mechanisms. While the C-terminal kinase catalytic domain is a region of high homology among all human PAKs, the N-terminal regions are more variable. PAKs belonging to group I (isoforms 1, 2 and 3) contain a con-

\footnotetext{
$\square$ Corresponding author: Joanna Szczepanowska, Laboratory of Bioenergetics and Biomembranes, Nencki Institute of Experimental Biology, Polish Academy of Sciences, L. Pasteura 3, 02-093 Warszawa, Poland; phone: (48) 22 589 2345; fax: (48) 22822 5342; e-mail: j.szczepanowska@nencki.gov.pl

Abbreviations: AD, Alzheimer's disease; ALS, amyotrophic lateral sclerosis; ALS2, alsin; BAD, BCL2 antagonist of cell death; CaD, caldesmon; CRIB, Cdc42/Rac-interacting binding domain; DLC1, dynein light chain; FLNa, filamin A; GEF, guanine exchange factor; GAP, GTPase activating protein; GC, guanylyl cyclase; GIT-1, G-protein coupled receptor kinase-interacting protein 1; ILK, integrin-linked kinase; Inca, induced in neuronal crest by activating protein; LIMK, LIM kinase; MARK, MAP/microtubule affinity-regulating kinase; MHC, myosin heavy chain; MLC, myosin light chain; MLCK, myosin light chain kinase; NET1, neuroepithelioma transforming gene 1; PAKs, p21-activated kinases; PIX, PAK-interactive exchange factor; p41-Arc (Arp2/3), 41 kDa subunit actin-related protein 2/3 complex; PRG, PDZ RhoGEF; p35/Cdk5, cyclin-dependent kinase 5 (Cdk5) and its neuron-specific regulator p35; RhoGDI1, Rho GDP dissociation inhibitor; RLC, regulatory light chain; $\mathrm{TCOB}$, tubulin cofactor $\mathrm{B}$.
} 
served Cdc42/Rac-interacting binding domain CRIB (also called p21-binding domain - PBD) and an autoinhibitory domain - AI. The N-terminal region of group II PAKs (isoforms 4, 5 and 6) also have a CRIB sequence, but lack a defined autoinhibitory domain (for references see: Jaffer \& Chernoff, 2002; Bokoch, 2003; Eswaran et al., 2008). Although both groups of PAKs specifically interact with Rho-family GTPases, PAKs of group I are activated upon binding Rac and Cdc42 whereas PAKs of group II do not require GTPases for their kinase activity, but their interaction with $\mathrm{Rac} / \mathrm{Cdc} 42$ affects the cellular localization of the protein. The mechanism of PAK activation has been studied in numerous organisms. The extensive and detailed information concerning the biochemical properties of PAKs and their regulation has been reviewed recently (for references see: Bokoch, 2003; Zhao \& Manser, 2005).

This review concentrates on the role of Rac/ Cdc42 regulated PAKs in maintaining and remodeling cytoskeletal structures in various organisms with a major focus on the newly identified PAK substrates that play an essential function in cytoskeletal rearrangements. Perturbations of the Rac/Cdc42/ PAK pathway leading to tumorigenesis and neurodegenerative diseases are also reviewed.

\section{PAKS ISOFORMS AND EXPRESSION PATTERN}

Members of the PAK family have been found in many organisms including Saccharomyces cerevisiae (Ste20, Cla4 and Skm1), Schizosaccharomyces pombe (Pak1p/Orb2p/Shk1p, Pak2p/Shk2p), Acanthamoeba castellani (MIHCK), Entamoeba histolytica (EhPAK2 and EhPAK3), Dictyostelium discoideum (DPAKa, DPAKb/MIHCK, DPAKc), Caenorhabditis elegans (CePAK1a, CeC45BII.1a, CeY38F1A), Drosophila melanogaster (DmPAK1, DmPAK3, Mbt/DmPAK2), Xenopus laevis (XIPAK1, 2, 3) and Homo sapiens (HsPAK1, 2, 3, 4, 5, 6) (Hofmann et al., 2004; Mentzel \& Raabe, 2005). The presence of PAK homologs in organisms ranging from protozoans to mammals strongly indicates ancient evolutionary origin and high importance of these proteins.

The pattern of mammalian PAKs expression has been studied for different cell types and tissues. For example, mammalian PAK1 is highly expressed in the brain, muscle and spleen, PAK 2 is expressed ubiquitously, PAK4 is present predominantly in the prostate, colon, and testis, PAK6 mainly in the testis, prostate and brain, while PAK3 and 5 are specifically expressed in the brain (Eswaran et al., 2008). The intracellular localization of a given PAK depends on its kinase activity, isoform and cell type. The binding partners of a PAK play a crucial role in its distribution in particular compartments. Activation of some PAKs changes their conformation allowing binding of new partners and resulting in different PAK distribution before and after its activation (for references see: Bokoch, 2003; Eswaran et al., 2008; Arias-Romero \& Chernoff, 2008).

\section{EFFECTS OF Rac/Cdc42/PAK PATHWAY ON CYTOSKELETAL ORGANIZATION}

The regulation of cytoskeletal organization by PAKs is very complex and involves both the C-terminal kinase catalytic domain and the N-terminal protein-protein interactions. The effects of PAK on the cytoskeleton can be dependent or independent of its kinase activity (Daniels \& Bokoch, 1999; AriasRomero \& Chernoff, 2008). Cdc42 and Rac are the predominant direct upstream signaling molecules of PAKs. PAK activated by Rac/Cdc42 induces formation of lamellipodia, filopodia, the membrane ruffles, stress fibres and remodeling of focal adhesion complexes (for references see: Bokoch, 2003). Proteins involved in the cytoskeletal reorganization include the main components of the actomyosin cytoskeleton, intermediate filaments, microtubules, integrins, and a variety of proteins associated with the above. PAK interacting with Rac/Cdc42 can modify properties of these proteins by their phosphorylation. It should be noted that PAK substrate specificity is similar among many species (Brzeska et al., 1997). The next chapter contains a list of substrates and binding partners of PAK directly engaged in cytoskeletal organization and those that are involved indirectly. The multiple regulatory roles of $\mathrm{PAK} / \mathrm{Rac} / \mathrm{Cdc} 42$ in cytoskeletal rearrangements are summarized in Fig. 1.

\section{Substrates and binding partners of PAKs directly implicated in cytoskeletal rearrangement}

Several of the PAK - interacting proteins are the main components of thin, thick and intermediate filaments. The myosin motor activity is crucial for the intracellular machinery responsible for cytoskeletal rearrangement implicated in spreading of the cell on a surface, cell migration and cell division, and myosins are targets of PAK.

Myosins constitute a large family of actindependent molecular motors that, upon interacting with actin, convert the chemical energy of ATP hydrolysis into mechanical movement. They consist of heavy (MHC) and light chains (MLC) and are present in all eukaryotic cells from yeast and amoeba to mammals. Myosins have been classified into nearly 30 classes (Sellers, 2000; Redowicz, 2007) based on the sequence homology of more than 250 known myosin motor domains. Class II myosins are called conventional myosins since they were first 


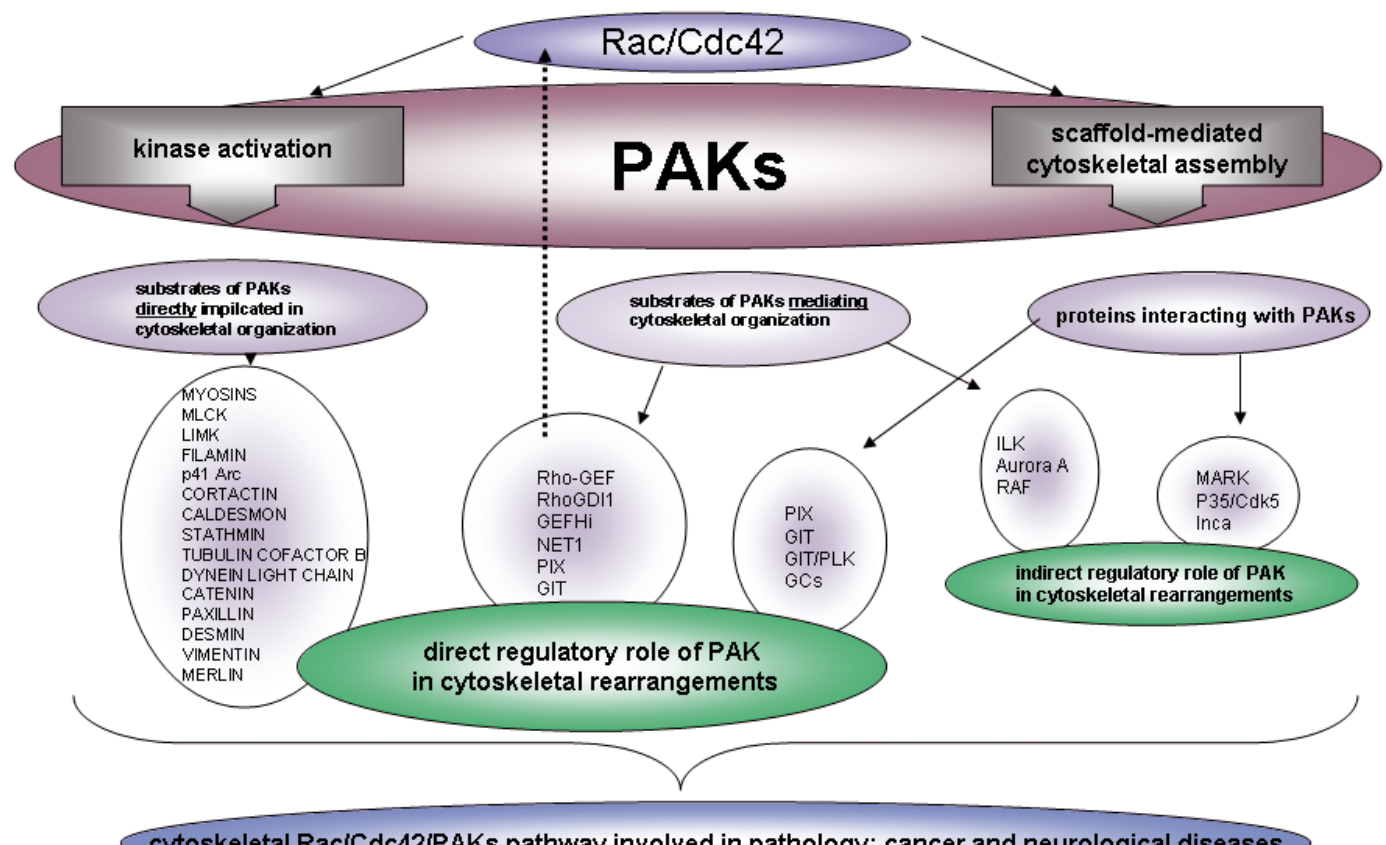

cytoskeletal RaciCdc42IPAKs pathway involved in pathology: cancer and neurological diseases

Figure 1. Involvement of Rac/Cdc42/PAK pathway in cytoskeletal rearrangements.

Substrates of PAKs and other proteins that interact with PAKs are indicated. Full names of the proteins are given in the text and in Abbreviations.

discovered. Myosins belonging to the other classes are called unconventional. Most of the conventional non-muscle myosins, as well as smooth muscle myosins II, are regulated by phosphorylation of their regulatory light chains (serine 19 and threonine 18 in smooth muscle myosin RLC). The activity of some myosins I is regulated by phosphorylation of the serine or threonine located in the actin-binding surface loop of the heavy chain (Brzeska \& Korn, 1996).

One of the first $\mathrm{Cdc} / \mathrm{Rac}$ effectors identified by Leberer et al. (1992) was Ste20 found in Saccharomyces cerevisiae. The Ste20 kinases belong to the PAK family and modulate cell morphology and polarity. The budding yeast $S$. cerevisiae has two myosin-I isoforms encoded by the MYO3 and MYO5 genes. Serine 357 residue, an activatory phosphorylation site of Myo3p heavy chain (that is also conserved in Myo5p), was identified as a unique phosphorylation site for Ste20p and Cla4p (another member of PAK family) both in vivo and in vitro (Wu et al., 1997). Phosphorylation of myosin I is required for yeast budding and regulates rearrangements of actin cytoskeleton (Lechler et al., 2000). The PAK-related protein Pak1p/Orb2p found in Schizosaccharomyces pombe localizes to the actomyosin ring at the cell division site. Pak1p mediates phosphorylation of myosin II regulatory light chain (Rlc1p) at serine 35 and serine 36 in vivo and loss of Pak1p activity leads to defective coordination of mitosis and cytokinesis (Loo \& Balasubramanian, 2008).
Acanthamoeba PAK-MIHCK catalyses phosphorylation of MHC of three Acanthamoeba myosin I isoforms causing their activation (Brzeska et al., 1989; 1996). Interestingly, Acanthamoeba PAK was probably the first discovered, purified and characterized PAK, long before mammalian PAK became known (Pollard \& Korn, 1973). Also in Dictyostelium, DdPAKB activates myosin ID by phosphorylation of its heavy chain (Lee \& Côté, 1995; Lee et al., 1996). In addition, Acanthamoeba PAK phosphorylates the regulatory light chain (RLC) of non-muscle myosin in vitro and in vivo (Brzeska et al., 1997; Szczepanowska et al., 2006). Expression of the active catalytic domain of amoeba PAK in HeLa cells causes phosphorylation of endogenous myosin II (Brzeska et al., 2004). Biochemical studies in vitro have demonstrated that Drosophila melanogaster PAK phosphorylates the RLC of Drosophila nonmuscle myosin II on serine 21 and threonine 20, sites homologous, respectively, to serine 19 and threonine 18 in mammalian smooth muscle myosin RLC (Crawford et al., 2001). In Xenopus laevis, activated XIPAK1 phosphorylates the regulatory light chain (xMLC) of myosin II on threonine 18 and serine 19 and induces hyperphosphorylation of xMLC in vivo. The XIPAK activation is sufficient for apoptotic body formation, and it is strongly suggest that activation of myosin II is essential for this process (Bisson et al., 2003). Mammalian PAK phosphorylates MLC on serine 19 (Ramos et al., 1997; Chew et al., 1998; Van Eyk et al., 1998). MLC phosphorylation (by PAK1 and PAK3) in neuronal cells promotes 
dendric spine morphogenesis by local stabilization of the actin network (Zhang et al., 2005). In non-neuronal cells, phoshorylated MLC localizes to both the leading edge and the rear end of a migrating cell (Matsumura et al., 1998). In addition to phosphorylating the RLC of nonmuscle and smooth muscle myosins II, and heavy chain of myosins I, PAK also phosphorylates heavy chain of myosin VI. Myosin VI plays an important role in membrane trafficking and cell migration (Buss et al., 1998).

PAK can also indirectly regulate phosphorylation of myosin by myosin light chain kinase (MLCK). PAK1 phosphorylates MLCK, thereby decreasing its activity (Sanders et al., 1999). PAK2 activated by Cdc42 can phosphorylate MLCK on serines 439 and 991, inhibiting its activity and limiting the development of isometric tension in smooth muscle and non-muscle cells (Goeckeler et al., 2000).

Another substrate for PAK kinases is LIM kinase (LIMK). LIMK is a serine/threonine kinase involved in the regulation of actin polymerization and microtubule disassembly (Bernard, 2007). PAK1 and PAK4 phosphorylate LIMK on threonine 508 causing LIMK activation (Edwards et al., 1999; Dan et al., 2001). Activated LIM kinase catalyses phosphorylation of cofilin which acts as an actin capping and severing protein. Phosphorylation of cofilin suppresses its activity, which increases in the amount of cellular filamentous actin (Arber et al., 1998; Yang et al., 1998). Cofilin is postulated to drive cell protrusion and migration by affecting polymerization of actin filaments. The role of PAK1 in the induction of lamellipodia, filopodia and formation of membrane ruffles as well as in cell motility involves LIMK phosphorylation (Bokoch, 2003). Moreover, PAK1 and PAK4 kinase activation and phosphorylation of LIMK is important for neuronal polarization and differentiation (Kreis \& Barnier, 2009).

Filamin A (FLNa) is an actin-binding protein that crosslinks actin filaments into orthogonal networks, and links them to cellular membranes. FLNa is phosphorylated on serine 2152 by PAK1. Filamin is required for PAK1-mediated actin changes and stabilization of membrane ruffling that occurs at the leading edge of motile cells. Moreover, FLNa binds to the CRIB region of PAK1 and stimulates PAK1 kinase activity, which indicates that this interaction is important for the local activation of PAK (Vadlamudi et al., 2002).

p41-Arc (Arp2/3) (41 kDa subunit actin-related protein 2/3 complex) is one of Arp2/3 components which have a regulatory role in the assembly and maintenance of the Arp2/3 complex. This complex is required for formation of branched networks of actin filaments at the cell cortex. The Arp2/3 complex consists of seven subunits. Phosphorylation of p41Arc on threonine 21 by PAK regulates its association with the Arp2/3 complex in the cortical actin nucleation regions of the cell. Moreover, interaction of PAK1 with p41-Arc has an important role in mammalian cell migration and may regulate cell motility and invasiveness (Vadlamudi et al., 2004b).

Cortactin is an F-actin binding protein, which is involved in actin polymerization and modulates formation of membrane ruffles, lamellipodia and podosomes. Phosphorylation of cortactin by PAK3 on serine 113 residue regulates actin polymerization and branching (Webb et al., 2006a; Ayala et al., 2008).

Caldesmon $(\mathrm{CaD})$ is an actin filament regulatory protein, and one of the key regulators of actin dynamics. In the smooth muscle caldesmon controls actin-myosin interactions. Mass spectroscopy data show that PAK phosphorylates $\mathrm{CaD}$ at serines 657 and 687 (Foster et al., 2000). PAK-catalyzed phosphorylation of $\mathrm{CaD}$ is involved in $\mathrm{Ca}^{2+}$-independent smooth muscle contraction. Caldesmon is localized to cell adhesion structures - podosomes. Mutations of caldesmon phosphorylation sites (by PAK1 and PAK2) inhibits cell polarization and leads to defects in membrane extension and cell migration (Eppinga et al., 2006; Morita et al., 2007).

Stathmin, also called oncoprotein 18 (Op18) is a microtubule-destabilizing protein which binds tubulin dimers, inhibits tubulin polymerization and promotes dynamic instability of microtubules. PAK1 phosphorylates Op18/stathmin specifically at serine 16. Phosphorylation inactivates this protein, which results in stabilization of microtubules at the leading edge of motile cells (Wittmann et al., 2004).

A cofactor in the assembly of $\alpha / \beta$-tubulin heterodimers - tubulin cofactor $\mathrm{B}(\mathrm{TCOB})$ - is a substrate of PAK1 in vivo as well as in vitro. PAK1 phosphorylates $\mathrm{TCOB}$ on serines 65 and 128 and colocalizes with $\mathrm{TCoB}$ on newly polymerized microtubules and at centrosomes. This phosphorylation of TCoB plays a role in the growth of new microtubules (Vadlamudi et al., 2005).

Dynein light chain (DLC1) is a component of the cytoplasmic dynein complex which moves along microtubules. Dynein is a motor for the retrograde transport of membranous organelles. This intracellular transport is fundamental to cellular morphogenesis and cellular functioning. PAK1 phosphorylation of DLC1 on serine 88 controls vesicle formation and trafficking functions (Vadlamudi et al., 2004a; Yang et al., 2005).

$\beta$-Catenin (homolog in Drosophila melanogaster - Armadillo) is linked directly or indirectly to the actin cytoskeleton via cadherins and $\alpha$-catenin. It is a multifunctional protein which regulates both cell-cell adhesion and nuclear transcription. Membrane-localized $\beta$-catenin is mostly bound at adherens junctions that maintain cell-cell contact, polar- 
ity and communication. In D. melanogaster activated Mbt (DmPAK2) is recruited to adherens junctions and phosphorylates Armadillo on Ser 561 and 688. These serine residues are conserved in vertebrate $\beta$ catenins. Activation of Mbt leads to destabilization of the interaction of Armadillo with cadherin resulting in a reduction of cadherin-mediated adhesion (Menzel et al., 2008).

Paxillin is an adaptor protein which mediates formation of integrin-dependent focal adhesions. Paxillin $\alpha$ isoform directly binds to, and is phosphorylated on serine by PAK3 activated by Cdc42. This indicates that paxillin serves as a link between PAK3 and focal adhesions (Hashimoto et al., 2001).

Proteins engaged in the organization of intermediate filaments which are substrates for PAKs are desmin and vimentin. Desmin is expressed in muscle cells, where it plays a critical role in maintaining the structural and functional integrity of myofibers. PAK1 phosphorylates desmin mainly on serine residues located in the head domain of this protein. Phosphorylation affects organization of desmin filaments and inhibits its ability to bind intermediate filaments (Ohtakara et al., 2000). Vimentin is one of the most widely expressed intermediate filament proteins. PAK1 regulates the organization of vimentin filaments through direct phosphorylation of this protein on serines 25, 38, 50, 65 and 72 (Goto et al., 2002).

Merlin tumor suppressor also called schwannomin is a member of a superfamily of membranecytoskeleton linking proteins - ERM. Proteins of this superfamily bind F-actin and modify actin polymerization. Merlin also interacts with many other proteins directly involved in cytoskeletal organization, such as ezrin, radixin, moesin, paxillin, $\mathrm{N}$ WASP, spectrin, tubulin, calpain and many others (a total of 34 interacting proteins have been identified so far) (Scoles, 2008). PAK phosphorylates merlin on serine 518, and this phosphorylation results in inhibition of merlin tumor suppressing activity and its translocation to the plasma membrane in a paxillin-dependent manner (Thaxton et al., 2007). Phosphorylation stabilizes this protein in a conformation described as an open state and unmasks binding sites for transmembrane receptors and some actin-associated proteins such as $\beta 1$ integrin and paxillin (Thaxton et al., 2008).

Rho GTPases play a key role in actin cytoskeleton remodeling. Their activity is regulated by guanine exchange factors (GEFs) and by GTPase activating proteins (GAPs). PDZ RhoGEF (PRG) is a GEF. The activity of PRG increases upon binding to heterotrimeric $G$ proteins. PAK4 associates with and phosphorylates PRG thus negatively regulating Rho activity. Therefore, it has been suggested that PAK4 provides a novel cross-talk mechanism between different GTPases (Barac et al., 2004).

Rho GDP dissociation inhibitor (RhoGDI1) negatively regulates the activity of Rho GTPases. PAK1 phosphorylates RhoGDI1 on serines 110 and 174, leading to activation of Rac1 (DerMardirossian et al., 2004). PAK2 binds to and phosphorylates RhoGDI1 on serines 34 and 101 (both in vitro and in vivo). This phosphorylation results in activation of Rac1/Cdc42 GTPases during neurite outgrowth (Shin et al., 2009).

GEF-H1 is another guanine nucleotide exchange factor controling Rho activity. PAK1 phosphorylates in vivo GEF-H1 on serine 885. Phosphorylated GEF-H1 binds $14-3-3$ protein causing its translocation to microtubules. This phosphorylation may also coordinate Rho, Rac and Cdc42-mediated signaling pathways (Zenke et al., 2004). PAK4 forms a stable complex with GEF-H1 and phosphorylates it on serines 67 and 810 . Phosphorylation of both sites plays an important role in controlling the localization and function of GEF-H1. The activity of GEF-H1 plays a role in the crosstalk between actin and microtubules networks, which is crucial for cell morphology and motility (Callow et al., 2005).

PAK1 also regulates the activity of RhoA-specific guanine nucleotide exchange factor - NET1 (neuroepithelioma transforming gene 1). PAK-dependent phosphorylation of NET1 on serines 152, 153 and 538 reduces its activity as a GEF and stimulates actin stress fiber formation (Alberts et al., 2005).

Integrin-linked kinase (ILK) is a major signaling integrator in mammalian cells. It plays a critical role in cell motility, cytoskeleton reorganization and tumor progression and invasion. PAK1 phosphorylates ILK on threonine 173 and serine 246 in vitro and in vivo (Acconcia et al., 2007). Mutation of these phosphorylation sites inhibited cell growth and migration.

\section{Other PAK substrates indirectly invoved in cy- toskeletal rearrangements}

Among the great number of molecules which are substrates of PAK kinase, some of them are indirectly implicated in cytoskeletal organization. Also many of proteins are indirectly involved in cytoskeletal rearrangements only by their interaction with a PAK molecule.

PIX (PAK-interactive exchange factor) also called COOL-1 is a specific guanine nucleotide exchange factor. Binding to PIX activates PAK and influences its localization (Rennefahrt et al., 2007; Manser et al., 1998). PAK1 phosphorylates $\beta$ PIX on serines 340 and 525 (Shin et al., 2002; Ballif et al., 2004; Olsen et al., 2006). Activated PAK and PIX translocate from focal complexes to cell-cell contact 
sites during wound closure (Zegers et al., 2003). In addition to PAK binding, PIX also binds to synaptic adaptor protein GIT/PKL (G protein-coupled receptor kinase-interacting protein/paxillin kinase linker). Interactions between GIT1 and PAK3/PIX/Rac play a particularly important role during spine morphogenesis and in mental retardation (Zhang et al., 2005). In this case, activated PAK (in a signaling complex consisting of GIT1, PIX, and Rac) acts through phosphorylation of myosin II regulatory light chain (MLC). Phosphorylated and activated MLC causes an increase in dendritic spine and synapse formation (Zhang et al., 2005). Another type of interaction between PIX and PAK was found in a mouse macrophage-like cell line (RAW274) during chemotaxis. Subunits released from heterotrimeric $G$ proteins $(\beta \gamma)$ bind to PAK1 and indirectly cause its activation through $\alpha$-PIX and Cdc42. This G $\beta \gamma /$ PAK1/PIX/ Cdc42 pathway influences localization of F-actin at the leading edge of the cell and regulates directional cell migration during chemotaxis (Li et al., 2003).

GIT-1, G-protein coupled receptor kinase-interacting protein 1 is GAP of Arf protein that binds PIX and paxillin. PAK1 phosphorylates GIT1 in vitro on serine 517 (Zhao et al., 2005) and on serine 709 (Webb et al., 2006b). GIT1 phosphorylation on serine 709 increases its binding to paxillin and is necessary for a GIT-dependent increase in protrusive activity. The PAK/PIX/GIT1/paxillin signaling pathway has a role in regulating focal adhesion turnover. PAK, PIX and GIT also have another function, they participate in regulation of centrosome maturation (Zhao et al., 2005).

Activation of the cell cycle normally requires cytoskeletal remodeling involving cooperation and coordination of numerous cellular events. PAKs are indirectly engaged in cell cycle regulation via their involvement in microtubule network rearrangement and formation of microtubule organizing center. Aurora-A (known as centrosomal-located kinase) is involved in cell cycle. Only activated PAK1 can bind to Aurora-A at the centrosome. PAK1 phosphorylates Aurora-A on threonine 288 and serine 342, and this phosphorylation is responsible for Aurora kinase activation in mitosis. Inhibition of PAK (or $\beta$ PIX depletion) causes delay in centrosome maturation (Zhao et al., 2005).

Raf family protein kinases are key effectors of Ras-mediated adhesion-dependent signaling that are also implicated in cytoskeletal organization. PAK1-3 phosphorylate Raf- 1 on serine 338 in vivo and in vitro and this phosphorylation stimulates the kinase activity (King et al., 1998).

Proteins interacting with PAKs and indirectly implicated in cytoskeletal rearrangements

MARK (MAP/microtubule affinity-regulating kinase) is involved in establishing embryonic polar- ity. In neurons, MARK phosphorylates tau protein (microtubule associated protein) thus destabilizing microtubules. PAK5 binds to and inhibits the activity of MARK2, which affects microtubule dynamics (Matenia et al., 2005; Timm et al., 2006).

Cyclin-dependent kinase $5(\mathrm{Cdk} 5)$ and its neuron-specific regulator p35 are essential for neuron migration. p35/Cdk5 concentrates at the leading edges of axonal growth cones and regulates neurite outgrowth. PAK1 is present in the Rac-p35/Cdk5 complexes. Active p35/Cdk5 kinase causes PAK1 hyperphosphorylation and affects the reorganization of the actin cytoskeleton in neurons, thus promoting neuronal migration and neurite outgrowth (Nikolic et al., 1998; Banerjee et al., 2002). Moreover, p53/Cdk5 phosphorylates PAK1 in cells undergoing mitosis. Activated PAK1 localizes to microtubule-organizing centers and along parts of the spindles and causes microtubules lengthening (Banerjee et al., 2002).

PAKs are also implicated in the guanylyl cyclases (GC) pathway. GCs catalyze the conversion of GTP to cGMP. cGMP is a ubiquitous second messenger mediating cellular responses to various exogenous and endogenous signaling molecules. Transmembrane GCs are directly stimulated by PAK kinases. Activation of GCs leads to increased cellular cGMP levels. Rac-PAK-GC signaling is important for the formation of lamellipodia (Guo et al., 2007).

Inca (induced in neuronal crest by activating protein) is a newly identified protein required for morphogenesis of the neural crest (NC). Xenopus PAK5 binds to and cooperates with Inca in restructuring cytoskeletal organization and the regulation of cell adhesion in the early embryo and in neural crest cells during craniofacial development. Mammalian PAK4 binds Inca thereby reorganizing the cytoskeleton and directing cell adhesion during craniofacial development (Luo et al., 2007).

\section{IMPLICATIONS OF PAKs IN PATHOPHYSIOLOGY}

Deregulation of PAKs has been reported in several human tumors and neurodegenerative diseases. Particularly the Rac/Cdc42/PAKs pathway, which is mainly implicated in cytoskeletal rearrangement, is involved in complex processes such as carcinogenesis (tumorigenesis) and neurodegeneration.

\section{Cancerogenesis}

PAKs play an important role in numerous types of tumor (for references see: Vadlamudi \& Kumar, 2003; Kumar et al., 2006). A crucial step in tumor progression is rearrangement of the cytoskeleton which is necessary for cell migration and inva- 
sion. Cell movement requires a defined cell polarity, membrane protrusion at the leading edge, adhesion and retraction of the rear end of the cell. Because PAKs regulate cytoskeletal organization, their overexpression or dysfunction of the Rac/Cdc42/PAK pathway in tumor cells can potentially alter cytoskeletal remodeling. PAK1, 4 and 6 are the only PAK family members that are directly oncogenic (Callow et al., 2002; Kumar et al., 2006), and expression of these proteins has been shown to be increased in a variety of cancer cell lines. PAK1 is overexpressed in ovarian and breast cancers, bladder transitional-cell carcinoma, T-cell lymphoma and glioblastomas (Kumar \& Vadlamudi, 2002). Moreover, overexpression of PAK4 and PAK6 as well as hyperactivation of PAK2 have been reported in prostate cancer (Kumar et al., 2006). In addition, PAK1, PAK2 and PAK4 are activated by cellular cues which stimulate cell migration. PAK1 and 2 cooperate in a carcinoma cell line to ensure optimal focal adhesion generation and maturation during migration (Coniglio et al., 2008). In breast cancer cells, expression of active PAK1 results in abnormal organization of the mitotic spindle characterized by the appearance of multiple spindle orientation (Vadlamudi \& Kumar, 2003). A PAK1 mutant in the highly invasive breast cancer cells causes enhanced cell spreading, stabilization of stress fibres and reduced invasiveness. Activated PAK1 can also promote cancer progression by phosphorylation and inactivation of a proapoptotic protein - BAD (BCL2 antagonist of cell death) - thereby inhibiting the proapoptotic effects of BAD (Schürmann et al., 2000). The presence of activated forms of PAK1 in the cytoplasm of glioblastoma cells is correlated with shorter survival of patients probably due to enhanced invasiveness of these cancer cells (Aoki et al., 2007). PAK4 directly interacts with an integrin cytoplasmic subunit and has an effect on cell motility in an integrin-specific manner in breast carcinoma cells (Zhang et al., 2002).

PAKs are also involved in genetic disorders responsible for tumors of the central and peripheral nervous system. PAK1 mediates phosphorylation and inactivation of Merlin, which may play a role in tumor cell spreading and metastasis (Kumar et al., 2006). Rac/Cdc42/PAK pathway regulates many key cellular processes which are affected during tumor development and metastasis. Many substrates of PAK play an indirect roles in this process. LIMK has been found to be overexpressed in breast and prostate tumors. p41-ARC phosphorylated by PAK mediates actin nucleation and filament assembly and regulates invasiveness of breast cancer cells. Another PAK substrate, stathmin, is overexpressed in several malignancies, and TCOB upregulation has been found in human breast tumors (Kumar et al., 2006). The Rac/Cdc42/PAKs pathway is also implicated in modulation of endothelial cell motility and morphology in tumor angiogenesis (Fryer \& Field, 2005).

\section{Neurodegenerative diseases}

$\mathrm{AD}$ - Alzheimer's disease. Alzheimer is the most often occurring human progressive neurodegenerative disorder. It is characterized by accumulation of $\beta$-amyloid protein aggregates. This accumulation causes abnormal spine morphology and synaptic defects like synaptic loss and synaptic dysfunction (Small, 2008). PAK 1, 2 and 3 are important regulators of synaptic plasticity, because it depends on the actin cytoskeletal organization in dendric spines. Some studies show that $\beta$-amyloid oligomers inhibit PAK, and this may result in disassembly of synaptic actin filaments. Recent data suggest that the loss of neurons and synapses observed in $\mathrm{AD}$ is related to deregulation of PAK1 and 3 expression and enzymatic activities (Nguyen et al., 2008; Salminen et al., 2008).

ALS - amyotrophic lateral sclerosis. A neurite outgrowth defect is associated with some subtypes of amyotrophic lateral sclerosis disease. Although the etiology of ALS is not fully understood, it seems that at least the juvenile form of this disease is caused by a mutation in alsin (ALS2), which is a GEF for Rac. PAK1 activated by alsin/Rac promotes neurite extension in hippocampal neuron growth cones, thus an impairment of the alsin/Rac/PAK1 pathway may contribute to motor neuron disease (Kreis \& Barnier, 2009).

Huntington disease. Aggregation of a protein called huntingtin is implicated in the development of this neurodegenerative disease. PAK1 binds to huntingtin in vitro and in vivo, leading to an enhanced oligomerization of this protein (Luo et al., 2008).

\section{Mental retardation}

PAK3 plays a specific role in synaptic plasticity, and is implicated in dendritic spine morphogenesis. Five mutations affecting different domains in the PAK3 molecule that correlate with mental retardation have been identified so far (for references see: Kreis \& Barnier, 2009). Three mutations associated with mental retardation have different effects on the biological functions of PAK3. Two of them completely abrogate the kinase activity, and the third one decreases the binding of PAK3 to Cdc42 and impairs PAK activation. Expression of kinase"dead" mutants of PAK3 alters spine morphology, whereas expression of a mutant with impaired activation by Cdc42 drastically decreases spine density. These data indicate that Cdc42/PAK3 participates in dendritic spine formation and synaptic plasticity (Kreis et al., 2007). Overexpression of constitutively 
active PAK3 also potently rescues abnormal spine morphology caused by mutation of $\alpha$ PIX (NodéLanglois et al., 2006).

\section{CONCLUSIONS}

All the data presented here show that the Rac/Cdc42/PAK pathway plays a fundamental role in the regulation of the cytoskeleton in a variety of organisms by phosphorylation and interaction with numerous proteins implicated directly and indirectly in cytoskeletal rearrangements (Fig. 1). This in turn implies that PAKs may ultimately be linked to various pathologies, including neuronal degeneration, cancer, and mental retardation. Therefore, understanding of the molecular mechanisms responsible for PAK activation and regulation as well as identification of their multiple interacting partners and kinase substrates seems to be very important.

\section{REFERENCES}

Acconcia F, Barnes CJ, Singh RR, Talukder AH, Kumar R (2007) Phosphorylation-dependent regulation of nuclear localization and functions of integrin-linked kinase. Proc Natl Acad Sci USA 104: 6782-6787.

Alberts AS, Qin H, Carr HS, Frost JA (2005) PAK1 negatively regulates the activity of the Rho exchange factor NET1. J Biol Chem 280: 12152-12161.

Aoki H, Yokoyama T, Fujiwara K, Tari AM, Sawaya R, Suki D, Hess KR, Aldape KD, Kondo S, Kumar R, Kondo Y (2007) Phosphorylated Pak1 level in the cytoplasm correlates with shorter survival time in patients with glioblastoma. Clin Cancer Res 13: 6603-6609.

Arber S, Barbayannis FA, Hanser H, Schneider C, Stanyon CA, Bernard O, Caroni P (1998) Regulation of actin dynamics through phosphorylation of cofilin by LIM-kinase. Nature 393: 805-809.

Arias-Romero LE, Chernoff J (2008) A tale of two Paks. Biol Cell 100: 97-108.

Ayala I, Baldassarre M, Giacchetti G, Caldieri G, Tetè S, Luini A, Buccione R (2008) Multiple regulatory inputs converge on cortactin to control invadopodia biogenesis and extracellular matrix degradation. J Cell Sci 121: 369-378.

Ballif BA, Villén J, Beausoleil SA, Schwartz D, Gygi SP (2004) Phosphoproteomic analysis of the developing mouse brain. Mol Cell Proteomics 3: 1093-1101.

Banerjee M, Worth D, Prowse DM, Nikolic M (2002) Pak1 phosphorylation on T212 affects microtubules in cells undergoing mitosis. Curr Biol 12: 1233-1239.

Barac A, Basile J, Vázquez-Prado J, Gao Y, Zheng Y, Gutkind JS (2004) Direct interaction of p21-activated kinase 4 with PDZ-RhoGEF, a G protein-linked Rho guanine exchange factor. J Biol Chem 279: 6182-6189.

Bernard O (2007) Lim kinases, regulators of actin dynamics. Int J Biochem Cell Biol 39: 1071-1076.

Bisson N, Islam N, Poitras L, Jean S, Bresnick A, Moss T (2003) The catalytic domain of xPAK1 is sufficient to induce myosin II dependent in vivo cell fragmentation independently of other apoptotic events. Dev Biol 263: 264-281.
Bokoch GM (2003) Biology of the p21-activated kinases. Annu Rev Biochem 72: 743-781.

Brzeska H, Korn ED (1996) Regulation of class I and class II myosins by heavy chain phosphorylation. J Biol Chem 279: 16983-16986.

Brzeska H, Lynch TJ, Martin B, Korn ED (1989) The localization and sequence of the phosphorylation sites of Acanthamoeba myosin I. An improved method for locating the phosphorylated amino acid. J Biol Chem 264: 19340-19348.

Brzeska H, Szczepanowska J, Hoey J, Korn ED (1996) The catalytic domain of acanthamoeba myosin 1 heavy chain kinase II. Expression of active catalytic domain and sequence homology to p21-activated kinase (PAK). J Biol Chem 271: 27056-27062.

Brzeska H, Knaus UG, Wang ZY, Bokoch GM, Korn ED (1997) p21-activated kinase has substrate specificity similar to Acanthamoeba myosin I heavy chain kinase and activates Acanthamoeba myosin I. Proc Natl Acad Sci USA 94: 1092-1095.

Brzeska H, Szczepanowska J, Matsumura F, Korn ED (2004) Rac-induced increase of phosphorylation of myosin regulatory light chain in HeLa cells. Cell Motil Cytoskeleton 58: 186-199.

Buss F, Kendric-Jones J, Lionne C, Knight AE, Cote GP, Luzio JP (1998) The localization of myosin VI at the Golgi complex and leading edge of fibroblasts and its phosphorylation and recruitment into membrane ruffles at A431 cells after growth factor stimulation. J Cell Biol 143: 1535-1545.

Callow MG, Clairvoyant F, Zhu S, Schryver B, Whyte DB, Bischoff JR, Jallal B, Smeal T (2002) Requirement for PAK4 in the anchorage-independent growth of human cancer cell lines. J Biol Chem 277: 550-558.

Callow MG, Zozulya S, Gishizky ML, Jallal B, Smeal T (2005) PAK4 mediates morphological changes through the regulation of GEF-H1. J Cell Sci 118: 1861-1872.

Chew TL, Masaracchia RA, Goeckeler ZM, Wysolmersky RB (1998) Phosphorylation of non-muscle myosin II regulatory light chain by p21-activated kinase (gammaPAK). J Muscle Res Cell Motil 19: 839-854.

Coniglio SJ, Zavarella S, Symons MH (2008) Pak1 and Pak2 mediate tumor cell invasion through distinct signaling mechanisms. Mol Cell Biol 28: 4162-4172.

Crawford JM, Su Z, Varlamova O, Bresnic AR, Kiehart DP (2001) Role of myosin-II phosphorylation in V12Cdc42mediated disruption of Drosophila cellularization. Eur J Cell Biol 80: 240-244.

Dan C, Kelly A, Bernard O, Minden A (2001) Cytoskeletal changes regulated by the PAK4 serine/threonine kinase are mediated by LIM kinase 1 and cofilin. J Biol Chem 276: 32115-32121.

Daniels RH, Bokoch GM (1999) p21-activated protein kinase: a crucial component of molecular signaling? Trends Biochem Sci 24: 350-355.

DerMardirossian C, Schnelzer A, Bokoch GM (2004) Phosphorylation of RhoGDI by Pak1 mediates dissociation of Rac GTPase. Mol Cell 15: 117-127.

Edwards DC, Sanders LC, Bokoch GM, Gordon NG (1999) Activation of LIM-kinase by PAK1 couples Rac/Cdc42 GTPase signaling to actin cytoskeletal dynamics. Nat Cell Biol 1: 253-259.

Eppinga RD, Li Y, Lin JL, Mak AS, Lin JJ (2006) Requirement of reversible caldesmon phosphorylation at P21activated kinase-responsive sites for lamellipodia extensions during cell migration. Cell Motil Cytoskel 63: 543-562. 
Eswaran J, Soundararajan M, Kumar R, Knapp S (2008) UnPAKing the class differences among p21-activated kinases. Trends Biochem Sci 33: 394-403.

Foster DB, Shen LH, Kelly J, Thibault P, Van Eyk JE, Mak AS (2000) Phosphorylation of caldesmon by p21-activated kinase. Implications for the $\mathrm{Ca}^{2+}$ sensitivity of smooth muscle contraction. J Biol Chem 275: 1959-1965.

Fryer BH, Field J (2005) Rho, Rac, Pak and angiogenesis: old roles and newly identified responsibilities in endothelial cells. Cancer Lett 229: 13-23.

Goeckeler ZM, Masaracchia RA, Zeng Q, Chew TL, Gallagher P, Wysomerki RB (2000) Phosphorylation of myosin light chain kinase by p21-activated kinase PAK2. J Biol Chem 275: 18366-18374.

Goto H, Tanabe K, Manser E, Lim L, Yasui Y, Inagaki M (2002) Phosphorylation and reorganization of vimentin by p21-activated kinase (PAK). Genes Cells 7: 1-7.

Guo D, Tan YC, Wang D, Madhusoodanan KS, Zheng Y, Maack T, Zhang JJ, Huang XY (2007) A Rac-cGMP signaling pathway. Cell 128: 341-355.

Hashimoto S, Tsubouchi A, Mazaki Y, Sabe H (2001) Interaction of paxillin with p21-activated kinase (PAK). Association of paxillin alpha with the kinase-inactive and the Cdc42-activated forms of PAK3. J Biol Chem 276: 6037-6045.

Hofmann C, Shepelev M, Chernoff J (2004) The genetics of PAK. J Cell Sci 117: 4343-4354.

Jaffe AB, Hall A (2005) Rho GTPases: biochemistry and biology. Annu Rev Cell Dev Biol 21: 247-269.

Jaffer ZM, Chernoff J (2002) p21-activated kinases:three more join the PAK. Int J Biochem Cell Biol 34: 713-717.

King AJ, Sun H, Diaz B, Barnard D, Miao W, Bagrodia S, Marshall MS (1998) The protein kinase PAK3 positively regulates Raf-1 activity through phosphorylation of serine 338. Nature 396: 180-183.

Knaus UG, Bokoch G (1998) The p21-activated kinase (PAKs). Int J Biochem Cell Biol 30: 857-862.

Kreis P, Barnier JV (2009) PAK signalling in neuronal physiology. Cell Signal 21: 384-393.

Kreis P, Thévenot E, Rousseau V, Boda B, Muller D, Barnier JV (2007) The p21-activated kinase 3 implicated in mental retardation regulates spine morphogenesis through a Cdc42-dependent pathway. J Biol Chem 282: 21497-21506.

Kumar R, Vadlamudi RK (2002) Emerging functions of p21-activated kinases in human cancer cells. J Cell Physiol 193: 133-144.

Kumar R, Gururaj AE, Barnes CJ (2006) p21-activated kinases in cancer. Nat Rev Cancer 6: 459-471.

Leberer E, Dignard D, Harcus D, Thomas DY, Whiteway M (1992) The protein kinase homologue Ate20p is required to link the yeast pheromone response G-protein beta gamma subunits to downstream signaling components. EMBO J 11: 4815-4824.

Lechler T, Shevchenko A, Li R (2000) Direct involvement of yeast type I myosins in Cdc42-dependent actin polymerization. J Cell Biol 148: 363-373.

Lee SF, Côté GP (1995) Purification and characterization of a Dictyostelium protein kinase required for actin activation of the $\mathrm{Mg}^{2+}$ ATPase activity of Dictyostelium myosin ID. J Biol Chem 270: 11776-11782.

Lee SF, Egelhoff TT, Mahasneh A, Côté GP (1996) Cloning and characterization of a Dictyostelium myosin I heavy chain kinase activated by Cdc42 and Rac. J Biol Chem 271: 27044-27048.

Li Z, Hannigan M, Mo Z, Liu B, Lu W, Wu Y, Smrcka AV, Wu G, Li L, Liu M, Huang CK, Wu D (2003) Directional sensing requires $G$ beta gamma-mediated PAK1 and PIX alpha-dependent activation of Cdc42. Cell 114: 215-227.

Loo T-H, Balasubramanian M (2008) Schizosaccharomyces pombe Pak-related protein, Pak1p/Orb2p, phosphorylates mosin regulatory light chain to inhibit cytokinesis. J Cell Biol 183: 785-793.

Luo T, Xu Y, Hoffman TL, Zhang T, Schilling T, Sargent D (2007) Inca: a novel p21-activated kinase-associated protein required for cranial neural crest development. Development 134: 1279-1289.

Luo S, Mizuta H, Rubinsztein DC (2008) p21-activated kinase 1 promotes soluble mutant huntingtin self-interaction and enhances toxicity. Hum Mol Genet 17: 895-905.

Manser E, Leung T, Salihuddin H, Zhao ZS, Lim L (1994) A brain serine/threonine protein kinase activated by Cdc42 and Rac1. Nature 367: 40-46.

Manser E, Loo TH, Koh CG, Zhao ZS, Chen XQ, Tan L, Tan I, Leung T, Lim L (1998) PAK kinases are directly coupled to the PIX family of nucleotide exchange factors. Mol Cell 1: 183-192.

Matenia D, Griesshaber B, Li X, Thiesses A, Johne C, Jiao J, Mandelkow E, Mandelkow E-M (2005) PAK5 kinase is an inhibitor of MARK/Par-1, which leads to stable microtubules and dynamic actin. Mol Biol Cell 16: 4410-4422.

Matsumura F, Ono S, Yamakita Y, Totsukawa G, Yamashiro S (1998) Specific localization of serine 19 phosphorylation myosin II during cell locomotion and mitosis in cultured cells. J Cell Biol 140: 119-129.

Mentzel B, Raabe T (2005) Phylogenetic and structural analysis of the Drosophila melanogaster p21-activated kinase DmPAK3. Gene 349: 25-33.

Menzel N, Melzer J, Waschke J, Lenz C, Wecklein H, Lochnit G, Dreckhahn D, Raabe T (2008) The Drosophila p21-activated kinase Mbt modulates DE-cadherin-mediated cell adhesion by phosphorylation of Armadillo. Biochem J 416: 231-241.

Morita T, Mayanagi T, Yoshio T, Sobue K (2007) Changes in the balance between caldesmon regulated by p21-activated kinases and the Arp2/3 complex govern podosome formation. J Biol Chem 282: 8454-8463.

Nguyen TV, Galvan V, Huang W, Banwait S, Tang H, Zhang J, Bredesen DE (2008) Signal transduction in Alzheimer disease: p21-activated kinase signaling requires C-terminal cleavage of APP at Asp664. J Neurochem 104: 1065-1080.

Nikolic M, Chou MM, Lu W, Mayer BJ, Tsai LH (1998) The p35/Cdk5 kinase is a neuron-specific Rac effector that inhibits Pak1 activity. Nature 395: 194-198.

Nodé-Langlois R, Muller D, Boda B (2006) Sequential implication of the mental retardation proteins ARHGEF6 and PAK3 in spine morphogenesis. J Cell Sci 119: 49864993.

Ohtakara K, Inada H, Goto H, Taki W, Manser E, Lim L, Izawa I, Inagaki M (2000) p21-activated kinase PAK phosphorylates desmin at sites different from those for Rho-associated kinase. Biochem Biophys Res Commun 272: 712-716.

Olsen JV, Blagoev B, Gnad F, Macek B, Kumar C, Mortensen P, Mann M (2006) Global, in vivo, and sitespecific phosphorylation dynamics in signaling networks. Cell 127: 635-648.

Pollard TD, Korn ED (1973) Acanthamoeba myosin. I. Isolation from Acanthamoeba castellanii of an enzyme similar to muscle myosin. J Biol Chem 248: 4691-4697.

Ramos E, Wysolmerski RB, Masaracchia RA (1997) Myosin phosphorylation by human cdc42-dependent S6/H4 kinase/gammaPAK from placenta and lymphoid cells. Recept Signall Transduct 7: 99-110. 
Redowicz MJ (2007) Unconventional myosins in muscle. Eur J Cell Biol 86: 549-558.

Rennefahrt UE, Deacon SW, Parker SA, Devarajan K, Beeser A, Chernoff J, Knapp S, Turk BE, Peterson JR (2007) Specificity profiling of Pak kinases allows identification of novel phosphorylation sites. J Biol Chem 282: 15667-15678.

Salminen A, Suuronen T, Kaarniranta K (2008) ROCK, PAK, and Toll of synapses in Alzheimer's disease. Biochem Biophys Res Commun 371: 587-590.

Sanders LC, Matsumura F, Bokoch GM, de Lanerolle P (1999) Inhibition of myosin light chain kinase by p21activated kinase. Science 283: 2083-2085.

Scoles DR (2008) The merlin interacting proteins reveal multiple targets for NF2 therapy. Biochim Biophys Acta 1785: 32-54.

Schürmann A, Mooney AF, Sanders LC, Sells MA, Wang HG, Reed JC, Bokoch GM (2000) p21-activated kinase 1 phosphorylates the death agonist Bad and protects cells from apoptosis. Mol Cell Biol 20: 453-461.

Sellers JR (2000) Myosins: a diverse superfamily. Biochim Biophys Acta 1496: 3-22.

Shin EY, Shin KS, Lee CS, Woo KN, Quan SH, soung NK, Kim YG, Cha CI, Kim SR, Park D, Bokoch GM, Kim EG (2002) Phosphorylation of p85 beta PIX, a Rac/Cdc42specific guanine nucleotide exchange factor, via the Ras/ERK/PAK2 pathway is required for basic fibroblast growth factor-induced neurite outgrowth. J Biol Chem 277: 44417-44430.

Shin EY, Shim ES, Lee CS, Kim HK, Kim EG (2009) Phosphorylation of RhoGDI1 by p21-activated kinase 2 mediates basic fibroblast growth factor-stimulated neurite outgrowth in PC12 cells. Biochem Biophys Res Commun 379: 384-389.

Small DH (2008) Neural network dysfunction in Alzheimer's disease: a drug development perspective. Trends Mol Med 14: 103-108.

Szczepanowska J, Korn ED, Brzeska H (2006) Activation of myosin in HeLa cells causes redistribution of focal adhesions and F-actin from cell center to cell periphery. Cell Motil Cytoskeleton 63: 356-374.

Thaxton C, Lopera J, Bott M, Baldwin ME, Kalidas P, Fernandez-Valle C (2007) Phosphorylation of the NF2 tumor suppressor in Schwann cells is mediated by Cdc42-Pak and requires paxillin binding. Mol Cell Neurosci 34: 231-242.

Thaxton C, Lopera J, Bott M, Fernandez-Valle C (2008) Neuregulin and laminin stimulate phosphorylation of the NF2 tumor suppressor in Schwann cells by distinct protein kinase A and p21-activated kinase-dependent pathways. Oncogene 27: 2705-2715.

Timm T, Matenia D, Griesshaber B, Mandelkow EM (2006) Signaling from MARK to tau: regulation, cytoskeletal crosstalk, and pathological phosphorylation. Neurodegener Dis 3: 207-217.

Vadlamudi RK, Kumar R (2003) P21-activated kinases in human cancer. Cancer Metastasis Rev 22: 385-393.

Vadlamudi RK, Li F, Adam L, Nguyen D, Ohta Y, Stossel TP, Kumar R (2002) Filamin is essential in actin cytoskeletal assembly mediated by p21-activated kinase 1. Nat Cell Biol 4: 681-690.

Vadlamudi RK, Bagheri-Yarmand R, Yang Z, Balasenthil S, Nguyen D, Sahin AA, den Hollander P, Kumar R (2004a) Dynein light chain 1, a p21-activated kinase 1interacting substrate, promotes cancerous phenotypes. Cancer Cell 5: 575-585.

Vadlamudi RK, Li F, Barnes CJ, Bagheri-Yarmand R, Kumar R (2004b) p41-Arc subunit of human Arp2/3 complex is a p21-activated kinase-1-interacting substrate. EMBO Rep 5: 154-160.

Vadlamudi RK, Barnes CJ, Rayala S, Li F, Balasenthil S, Marcus S, Goodson HV, Sahin AA, Kumar R (2005) p21-activated kinase 1 regulates microtubule dynamics by phosphorylating tubulin cofactor B. Mol Cell Biol 25: 3726-3736.

Van Eyk JE, Arrel DK, Foster DB, Strauss JD, Heininen TY, Furmaniak-Kazmierczak E, Cote GP, Mak AS (1998) Different molecular mechanisms for Rho family GTPase-dependent, $\mathrm{Ca}^{2+}$-independent contraction of smooth muscle. J Biol Chem 273: 23433-23439.

Webb BA, Zhou S, Eves R, Shen L, Jia L, Mak AS (2006a) Phosphorylation of cortactin by p21-activated kinase. Arch Biochem Biophys 456: 183-193.

Webb DJ, Mayhew MW, Kovalenko M, Schroeder MJ, Jeffery ED, Whitmore L, Shabanowitz J, Hunt DF, Horwitz AF (2006b). Identification of phosphorylation sites in GIT1. J Cell Sci 119: 2847-2850.

Wittmann T, Bokoch GM, Waterman-Storer CM (2004) Regulation of microtubule destabilizing activity of Op18/stathmin downstream of Rac1. J Biol Chem 279: 6196-6203.

Wu C, Lytvyn V, Thomas DY, Leberer E (1997) The phosphorylation site for Ste20p-like protein kinases is essential for the function of myosin-1 in yeast. $J$ Biol Chem 272: 30623-30626.

Yang N, Higuchi O, Ohashi K, Nagata K, Wada A, Kangawa K, Nishida E, Mizuno K (1998) Cofilin phosphorylation by LIM-kinase 1 and its role in Rac-mediated actin reorganization. Nature 393: 809-812.

Yang Z, Vadlamudi RK, Kumar R (2005) Dynein light chain 1 phosphorylation controls macropinocytosis. $J$ Biol Chem 280: 654-659.

Zegers MM, Forget MA, Chernoff J, Mostov KE, ter Beest MB, Hansen SH (2003) Pak1 and PIX regulate contact inhibition during epithelial wound healing. EMBO J 22: 4155-4165.

Zenke FT, Krendel M, DerMardirossian C, King CC, Bohl BP, Bokoch GM (2004) p21-activated kinase 1 phosphorylates and regulates 14-3-3 binding to GEF-H1, a microtubule-localized Rho exchange factor. J Biol Chem 279: $18392-18400$.

Zhang H, Li Z, Viklund EK, Strömblad S (2002) p21-Activated kinase 4 interacts with integrin $\alpha v \beta 5$ and regulates $\alpha v \beta 5$-mediated cell migration. J Cell Biol 158: 1287-1297.

Zhang H, Webb DJ, Assmunsen H, Niu S, Horwitz AF (2005) A GIT1/PIX/Rac/PAK signaling module regulates spine morphogenesis and synapse formation through MLC. J Neurosci 25: 3379-3388.

Zhao ZS, Lim JP, Ng YW, Lim L, Manser E (2005) The GIT-associated kinase PAK targets to the centrosome and regulates Aurora-A. Mol Cell 20: 237-249.

Zhao ZS, Manser E (2005) PAK and other Rho-associated kinases-effectors with surprisingly diverse mechanisms of regulation. Biochem J 386: 201-214. 